\title{
Symplectic Structure-Preserving Particle-in-Cell Whole-Volume Simulation of Tokamak Plasmas to 111.3 Trillion Particles and 25.7 Billion Grids
}

\author{
Jianyuan Xiao \\ Junshi Chen \\ xiaojy@ustc.edu.cn \\ cjuns@ustc.edu.cn \\ University of Science and \\ Technology of China \\ Hefei, China \\ Chao Yang \\ chao_yang@pku.edu.cn \\ School of Mathematical Sciences, \\ Peking University, Beijing, China
}

\author{
Yeqi Huang \\ Wenting Han \\ huangyeqi@mail.ustc.edu.cn \\ hwt@ustc.edu.cn \\ University of Science and \\ Technology of China \\ Hefei, China \\ Jiale Chen \\ Guoqiang Li \\ chen@ipp.cas.cn \\ ligq@ipp.ac.cn \\ Institute of Plasma Physics, Chinese \\ Academy of Sciences \\ Hefei, China
}

Jiangshan Zheng

zhengjs@mail.ustc.edu.cn

University of Science and

Technology of China

Hefei, China

Fang Li

38349735@qq.com

National Supercomputing Center in

Wuxi, Wuxi, China

\author{
Xin Liu* \\ Dexun Chen \\ yyylx@263.net \\ cdx15@mails.tsinghua.edu.cn \\ National Supercomputing Center in \\ Wuxi, Wuxi, China \\ Xuan Sun \\ xsun@ustc.edu.cn \\ University of Science and \\ Technology of China \\ Hefei, China
}

\author{
Hong $\mathrm{An}^{*}$ \\ Shenghong Huang* \\ han@ustc.edu.cn \\ hshnpu@ustc.edu.cn \\ University of Science and \\ Technology of China \\ Hefei, China \\ Ziyu Zhang \\ zzymm@mail.ustc.edu.cn \\ University of Science and \\ Technology of China \\ Hefei, China
Zixi Liu
Ge Zhuang
zxliu316@ustc.edu.cn
gezhuang@ustc.edu.cn
University of Science and
Technology of China
Hefei, China
Qiang Chen
qiangchen@zzu.edu.cn
Zhengzhou Uinversity
Zhengzhou, China

\begin{abstract}
We employ our recently developed explicit 2 nd-order chargeconservative symplectic electromagnetic particle-in-cell (PIC) scheme in the cylindrical mesh to simulate the whole-volume magnetic confinement toroidal plasmas on the new Sunway supercomputer. From a large-scale simulation of magneticized toroidal plasma with 111.3 trillion particles and 25.7 billion grids, we have obtained a sustained performance exceeding 201.1 PFLOP/s (double precision) with the fastest iteration step achieving 298.2 PFLOP/s (double precision). For

${ }^{*}$ Corresponding Authors

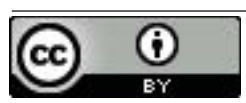

This work is licensed under a Creative Commons Attribution International 4.0 License. SC '21, November 14-19, 2021, St. Louis, MO, USA

(C) 2021 Copyright held by the owner/author(s).

ACM ISBN 978-1-4503-8442-1/21/11.

https://doi.org/10.1145/3458817.3487398
\end{abstract}

the first time, unprecedented high resolution evolution of 6D electromagnetic fully kinetic plasmas based on $2 \mathrm{D}$ equilibrium profiles from Experimental Advanced Superconducting Tokamak (EAST) and designed operation state of China Fusion Engineering Test Reactor (CFETR) are presented, and edge micro-instabilities can be investigated directly. This shows the possibility to study crucial problems and phenomena in the magnetic confinement toroidal plasma directly using the symplectic electromagnetic fully kinetic PIC method on world's leading supercomputers.

\section{ACM Reference Format:}

Jianyuan Xiao, Junshi Chen, Jiangshan Zheng, Hong An, Shenghong Huang, Chao Yang, Fang Li, Ziyu Zhang, Yeqi Huang, Wenting Han, Xin Liu, Dexun Chen, Zixi Liu, Ge Zhuang, Jiale Chen, Guoqiang Li, Xuan Sun, and Qiang Chen. 2021. Symplectic Structure-Preserving Particle-in-Cell Whole-Volume Simulation of Tokamak Plasmas to 111.3 Trillion Particles and 25.7 Billion Grids . In The International Conference for High Performance Computing, Networking, Storage and Analysis (SC '21), November 14-19, 2021, St. Louis, MO, 
USA. ACM, New York, NY, USA, 13 pages. https://doi.org/10.1145/3458817. 3487398

\section{JUSTIFICATION FOR ACM GORDON BELL PRIZE}

We employ our recently developed symplectic structure preserving fully kinetic Particle-in-Cell scheme in cylindrical coordinates and have measured $95.6 \%$ weak scaling efficiency and a sustained performance of 201.1 PFLOP/s using 103,600 nodes of the new Sunway supercomputer. Simulation of whole-volume tokamak plasma to $1.113 \times 10^{14}$ particles and $2.57 \times 10^{10}$ grids is achieved.

\section{PERFORMANCE ATTRIBUTES}

\begin{tabular}{ll}
\hline Category of achievement: & peak performance, scalability \\
Type of method used: & explicit symplectic \\
Results reported on the basis of: & whole application excluding I/O \\
Precision reported: & double precision \\
System Scale: & measured on full-scale system \\
Measurement Mechanism: & timers, FLOP count \\
\hline
\end{tabular}

\section{OVERVIEW OF THE PROBLEM}

\subsection{Development of Tokamaks}

Faced with the coming energy crisis, human beings have a common dream to seek an infinite and clean energy for sustainable development. Tokamak [2], initially conceptualized in the 1950s, is currently the most promising magnetic confinement fusion (MCF) device that can eventually provide clean, safe and nearly unlimited fusion energy in the future. Its basic idea is using strong toroidal magnetic field to confine high temperature plasmas. The magnetic field configuration of a Tokamak is shown in Fig. 1(a). Inside a Tokamak magnetic field, the particles are moving along the trapped orbit or passing orbit, and if no dissipation they will be bounded in the device permanently. Development of Tokamaks and the product they achieved is summarized in Fig. 1(b). It can be seen that inside a Tokamak which can actually output energy, the plasma needs to be heated to above 100 million degrees Kelvin in order to significantly exceed the fusion triple product condition, while the magnet surrounding the plasma requires very low temperature to maintain the superconductivity. Such huge temperature and pressure gradient will drive various micro-instabilities and turbulences that significantly degrade the confinement performance. To overcome this difficulty, the device is required to be very large, and international efforts have been made to build the next generation International Thermonuclear Experimental Reactor (ITER) [3]. Devices with similar plasma parameters, China Fusion Engineering Test Reactor (CFETR) [47] and SPARC [30], are also proposed for supplement of ITER and demonstration of power plant validation. Behaviours of fusion (burning) plasmas, energetic particles and how the high power auxiliary heating techniques designed for these large Tokamaks can effect the burning plasma are under investigation. While in another aspect, methods to suppress the turbulence are also worthy to be tried since they may decrease the cost. All these studies and attempts need high fidelity simulation of kinetic plasmas at extreme scales.

\subsection{Particle-in-Cell method applied to Tokamak physics}

The Particle-In-Cell (PIC) method proposed in 1960s $[6,13,19]$ is a powerful tool for studying plasmas and beam physics. It directly solves the evolution of charged particles coupled with electromagnetic fields, governed by the following equations:

$$
\begin{aligned}
\frac{\partial \ddot{\mathbf{x}}_{s p}}{\partial t} & =\frac{q_{s}}{m_{s}}\left(\mathbf{E}\left(\mathbf{x}_{s p}\right)+\dot{\mathbf{x}}_{s p} \times \mathbf{B}\left(\mathbf{x}_{s p}\right)\right), \\
\frac{\partial \mathbf{B}}{\partial t} & =-\nabla \times \mathbf{E}, \\
\frac{\partial \mathbf{E}}{\partial t} & =\nabla \times \mathbf{B}-\sum_{s, p} q_{s} \dot{\mathbf{x}}_{s p} \delta\left(\mathbf{x}-\mathbf{x}_{s p}\right),
\end{aligned}
$$

where dielectric constant and permeability in the vacuum are set to 1 for simplicity, $\mathbf{E}$ and $\mathbf{B}$ are electromagnetic fields, $q_{s}, m_{s}, \mathbf{x}_{s p}$ and $\dot{\mathbf{x}}_{s p}$ are the charge, mass, position and velocity for the $p$-th particle of species $s$. For many crucial but complex problems in magnetic confinement plasmas, such as the micro-instabilities in the edge plasma, lower hybrid current drive, ion cyclone resonant heating, and energetic particle induced instabilities, the first-principle based electromagnetic 6D (i.e., 3-dimensional space and 3-dimensional velocities) fully kinetic PIC method is desired, and usually these problems require simulations of whole-volume or near whole-volume plasmas. Understanding these problems can help explain experimental phenomena, design better plasma configuration and optimize auxiliary heating methods. This is especially useful for now because at present, modeling for next generation devices such as ITER and CFETR requires integrating higher-fidelity multiscale whole volume plasma models into simulations [7], as illustrated in Fig. 1(c). It will be significantly beneficial if we find a way by simulation that can reduce even $1 \%$ total cost of the devices.

In this work, we present large-scale fully kinetic simulations of whole-volume Tokamak plasmas using our recently developed explicit 2nd-order charge-conservative symplectic electromagnetic PIC scheme for cylindrical regular meshes [41]. For the first time, the whole-volume plasma based on equilibrium of existing Tokamak (e.g., Experimental Advanced Superconducting Tokamak, EAST [37]) experiment and designed operation state of future Tokamaks (e.g., CFETR) are numerically investigated directly using the most basic charged particle coupled with electromagnetic field plasma model. With the help of world's leading new Sunway supercomputer and good scalability of the scheme, we can simulate the evolution of unprecedented $N_{R} \times N_{\psi} \times N_{Z} \times \mathrm{NPG}=3072 \times 2048 \times$ $4096 \times 4320$ toroidal plasmas with $1.113 \times 10^{14}$ marker particles, where $N_{R}, N_{\psi}, N_{Z}, N_{\text {total }}$ and NPG are the number of grids in the axial, toroidal, height directions and number of marker particles per grid, respectively. A sustained performance of $3.724 \times 10^{13}$ particle push per second (201.1 PFLOP/s) and peak performance of one iteration step of $298.2 \mathrm{PFLOP} / \mathrm{s}$ are measured. As examples, we demonstrate the simulated high resolution of edge instabilities based on equilibrium profiles of EAST and designed CFETR H-mode plasmas.

\section{CURRENT STATE OF THE ART}

Due to the multiscale nature of the magnetic confinement plasmas, it is very difficult to apply the 6D PIC method for simulating 

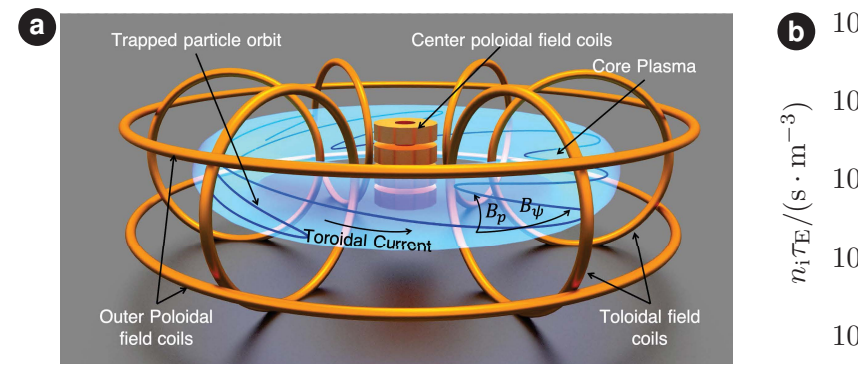

\begin{tabular}{|c|c|c|c|c|}
\hline Year & $1990 \mathrm{~s}$ & $2000 \mathrm{~s}$ & $2010 s$ & $2020 s$ \\
\hline Computing resource & GFlops & TFlops & PFlops & EFlops \\
\hline Spatial resolution & $\sim 40$ & $\sim 100$ & $\sim 250$ & $\sim 600$ \\
\hline Time range & Single & Single & Multiple & Multiple \\
\hline Physical model & MHD & $\begin{array}{c}2 \text {-fluid } \\
\text { Gyrokinetic }\end{array}$ & $\begin{array}{c}\text { 2-fluid } \\
\text { Gyrokinetic } \\
\text { Kinetic-MHD }\end{array}$ & $\begin{array}{l}\text { Gyrokinetic } \\
\text { Kinetic-MHD } \\
\text { Fully-Kinetic }\end{array}$ \\
\hline
\end{tabular}

Figure 1: The development of modern and future Tokamaks requires whole-volume simulations. (a) A schematic diagram of Tokamak magnet configuration and trapped particle orbit inside that magnetic field. (b) The achievement of fusion product of Tokamak devices, including 3 projection of planned devices (ITER, CFETR and SPARC) in the future, where $Q_{\mathrm{DT}}$ is the ratio between fusion power and heating power in a deuterium-tritium plasma. $Q_{\mathrm{DT}}>1$ means the fusion power is larger than the external heating power and $Q_{\mathrm{DT}}=\infty$ is the self-sustained burning condition or the well known Lawson criterion. Data are collected from Refs. [38, 44]. (c) The development of whole volume simulation of Tokamak plasmas, including the physical model, spatial-time range, and the computing resource available at that time. Designing and modeling of Tokamak operations with larger in size and higher parameters requires whole volume simulation of plasmas using high fidelity models that contains more physical processes.

Table 1: Performance of PIC simulators with different algorithms on top supercomputers. Here the abbreviations GK, FK, NPG, and CG represent gyrokinetic, fully kinetic and number of sampling particles per grid, and Sunway core group, respectively. Note that for GK PIC methods, solving GK Poisson or Ampere equations is usually implicit and may not have good scalability. The symplectic FK PIC scheme used in this work consumes about 5000 FLOPs when calculating the pushing and current deposition for one particle, while conventional non-symplectic Boris-Yee based FK PIC schemes this number is around 250 (VPIC [8])-650 (PIConGPU [45]) FLOPs.

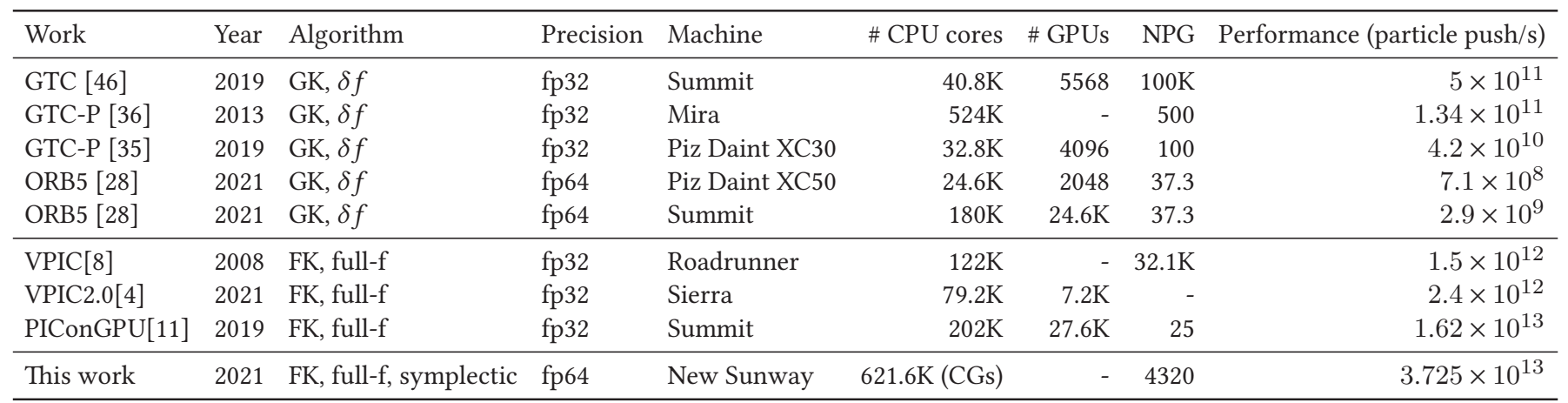

the evolution of whole-volume magnetic confinement plasmas. In this section, we introduce existing works focus on the PIC simulation on large-scale clusters, including gyrokinetic PIC simulation of toroidal plasmas and conventional 6D fully kinetic PIC simulations on top supercomputers. A summary including the present work is listed in Tab. 1.

\subsection{Gyrokinetic PIC simulations}

The space-time span of the whole-volume plasma evolution is very large, and directly using fully kinetic PIC schemes will cost huge amount of computational resources. To increase the maximum allowed time-step and hence reduce the computational cost, people introduced gyrokinetic (GK) PIC method [12, 15, 24, 25] in which 
the particles are modelled as guiding centers to investigate low frequency turbulence problems in Tokamaks [25]. It should be noted that in GK simulations not just the gyro-averaging approximation or gyro-center coordinate transform is adopted. Some other techniques such as the magnetic static approximation, parallel magnetic vector potential assumption, GK Poisson equation [24] and GK Ampere equation [15] for approximations of Maxwell equations are also included. This is because in strongly magnetized plasmas, not only the gyromotion, but also the plasma oscillation, electromagnetic modes, or even Alfvén modes will limit the time-step. GTC [46], GTC-P [35, 36] and ORB5 [28] are commonly used GK codes for large-scale plasma simulations on recently developed supercomputers. The performance behavior of GK PIC methods may be very different compared to fully kinetic PIC ones since they could involve implicit Poisson solvers, we still list them in Tab. 1 for reference.

In spite of the great success of gyrokinetic simulations, it was pointed out recently $[20,26]$ that the ordering assumption of the gyrokinetic theory $[14,23]$ is not always valid in certain circumstances [34]. Another issue is that solving Poisson equation in gyrokinetic codes does not scale well on large clusters, which may limit the parallel performance. These problems restrict the accuracy and speed of gyrokinetic simulations on modern large heterogeneous supercomputers.

\subsection{Fully kinetic PIC simulations}

Fully kinetic (FK) PIC methods directly solves the Vlasov-Maxwell or Vlasov-Poisson equations. Previously they were mainly used in simulating high-frequency physics such as the laser-plasma interactions and magnetic reconnections. A commonly used FK PIC scheme is the Boris-Yee scheme [5, 19], which is locally explicit and thus can have very good scalability on large clusters. A typical implementation, VPIC $[8,9]$, uses an improved version of this scheme. VPIC also employs a variety of Single Instruction Multiple Data (SIMD) intrinsics to fully utilize the floating point performance provided by modern processors. Recently people focus on porting conventional FK PIC schemes to GPU-based clusters, for example the development of VPIC2.0 [4] and PIConGPU [10, 11]. These FK electromagnetic PIC schemes are usually memory bandwidth bounded since one push and current deposition process in the Boris-Yee scheme only requires 250-650 floating point operations (FLOPs) $[8,45]$, while updating a particle in the memory need at least $24 / 48$ bytes read and $24 / 48$ bytes write for the case of fp32/fp64 precision.

\subsection{Symplectic PIC methods}

The fast development of supercomputers is making it possible to conduct 6D electromagnetic fully kinetic PIC simulations of wholevolume magnetic confinement plasmas. However, conventional 6D PIC algorithms do not posses the long-term accuracy and fidelity, which means even if unlimited computing resource exist, longterm results obtained by these algorithms are not reliable. To tackle this challenge, a series of structure preserving geometric PIC methods $[16,22,29,32,39-43]$ are developed to overcome the numerical dissipation (e.g., self-heating [18]) problem of conventional PIC methods. Based on discrete exterior calculus (DEC), Whitney interpolating form, canonical or noncanonical symplectic integrators, these PIC methods preserve discrete symplectic 2-form exactly during the iteration and thus have unparalleled long-term conservation property that conventional PIC methods do not have. Such conservation properties have been proved in practice $[17,22,27$, 43].

The symplectic structure-preserving PIC schemes are developed since 2012; however to our knowledge, the present work is the first one that investigates the performance optimizations and parallel scalability of the symplectic PIC simulation of whole-volume Tokamak devices on leadership supercomputers. Compared to the commonly employed Boris-Yee scheme, the 2nd-order charge-conservative symplectic PIC scheme used in this work requires more arithmetic operations for the push and current deposition process (about 5000 FLOPs according to our measurement) of one particle. So on most modern clusters the symplectic PIC scheme will become computationally intensive and more FLOP performance can be achieved. There are also other advantages to use symplectic PIC scheme in simulating magnetic confinement plasmas even if the memory bandwidth limitation on conventional Boris-Yee PIC schemes did not exist. First is that the grid size can be chosen to be much larger than the Debye length $\lambda_{\mathrm{D}}$ and no numerical instability is observed. Secondly the time-step can be also chosen as $\Delta t \Omega_{\mathrm{pe}} \sim 1$, while usually in conventional explicit PIC methods $\Delta t \Omega_{\mathrm{pe}}<0.2$ for the accuracy reason [33]. Lastly there is no numerical dissipation in the symplectic PIC scheme, which means we can run the simulation for very large number of time-steps as long as we need without worrying about the conservation property of the algorithm.

\section{INNOVATIONS REALIZED}

\subsection{Algorithm innovation}

In this work, we employ our recently developed state-of-the-art method, i.e., explicit 2nd-order charge conservative symplectic structure preserving electromagnetic PIC scheme for cylindrical coordinates. Detailed updating rule can be found in the Appendix B of Ref. [41]. It is based on modern geometric techniques such as DEC and Whitney interpolating forms in cylindrical coordinates, and variational symplectic or noncanonical Hamiltonian splitting integrators. Compared to conventional PIC schemes, our new symplectic PIC scheme exactly preserves the discrete noncanonical symplectic 2-form of the Hamiltonian charged particle-electromagnetic field system. Numerical errors of invariants such as total energy and momentum can be therefore bounded by a small number. As examples, the numerical self-heating problem in conventional PIC simulations is automatically eliminated in our symplectic PIC simulations, and the number of simulation time-steps can be choosen as much larger than that in conventional Boris-Yee based PIC schemes because no numerical dissipation is involved.

The algorithm is implemented in the SymPIC (https:/github.com/ JianyuanXiao/SymPIC/) which focuses on implementing structure preserving PIC schemes with both high performance and portability on modern large-scale heterogeneous clusters. The SymPIC code mainly consists of following modules: a scheme interpreter 
for loading configuration files, field solver, particle pusher and current deposition calculator, particle sorter, I/O module and the initializer for initial conditions. The workflow of SymPIC is shown in Fig. 2.

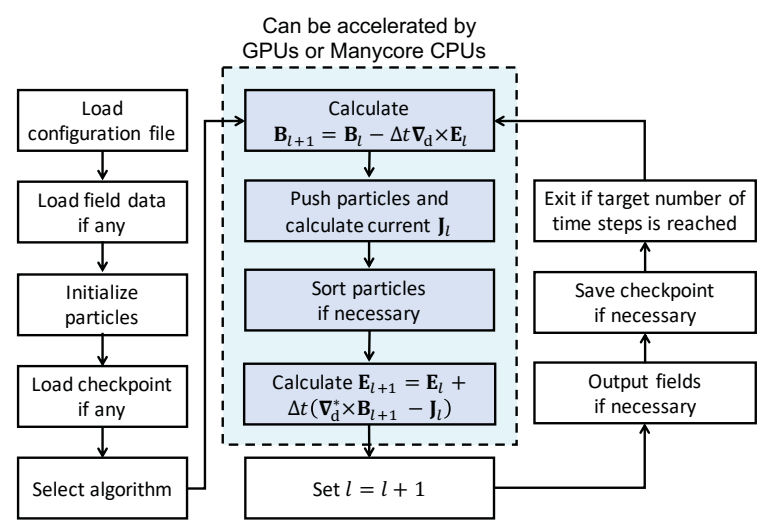

Figure 2: The workflow of SymPIC.

\subsection{Multi-platform code generation}

To increase the power efficiency and computing density, heterogeneous architectures are more preferable when building large supercomputing systems. Many of these heterogeneous architectures have their own programming APIs or even languages. For example, the Summit supercomputer utilizes an CPU+Nvidia GPU architecture, the Sunway TaihuLight supercomputer is built based on Sunway manycore processors, and the Tianhe-2A supercomputer employs an heterogeneous architecture with CPU+NUDT Matrix Accelerator. Developing applications to support all these heterogeneous platforms simultaneously could be challenging, but not impossible since all these heterogeneous cluster nodes have similar Management-Worker (MW) structures, therefore can be programmed with a consistent MW programming model. In particular, the conventional multi-core CPU can be also viewed as a special type of MW node.

To achieve multi-platform portability on heterogeneous clusters, we have developed a Domain Specific Language (DSL) named Parallel SCheme to Many Core (PSCMC, https://github.com/JianyuanX PSCMC) that can describe the MW programming model and corresponding code generator. It is Turing complete and hence can be employed to implement arbitrary parallel algorithms. Currently it supports code generation backends of serial C, OpenMP, CUDA, Sunway Manycore (SWMC) Athread interface, OpenCL, HIP, NUDT Matrix Accelerator Interface (MAI), and SYCL.

Our SymPIC code is implemented with PSCMC, therefore supports all the aforementioned parallel platforms, even including future CPU+AMD GPU and CPU+Intel GPU based clusters by using the PSCMC HIP and SYCL backends.

As an example, we demonstrate the multi-platform code generation procedure of the SymPIC code in Fig. 3. Firstly, some complex mathematical formulas used in SymPIC such as the particle pushing scheme and weighting functions are derived by using the Maxima computer algebra system [1] from the variation of discrete action integral as required by the symplectic method. This will make it easy to ensure the correctness of the tedious implementation of these complex formulas. Then the SymPIC PSCMC source code will be compiled into platform-specific code by using the PSCMC compiler, which is a multi-pass source-to-source compiler written in the scheme programming language. Its basic idea is dividing the compiler to a series of small source-to-source compiler passes, each of which performs a much easier task [21,31]. Some features such as a small type-inference system and a parallel-for loop vectorization procedure are also added.

Except for the good portability of codes to modern heterogeneous supercomputers, it also has many other advantages to use the PSCMC (or other similar DSLs, if any) for implementing numerical algorithms. Firstly, new MW heterogeneous platforms can be supported by adding corresponding code generation backends to PSCMC. According to our tests, to add support for one C programming language based heterogeneous programming environment such as OpenMP, CUDA, HIP, SWMC and MAI, only 100-200 lines of scheme code is required, which is significantly smaller compared to directly porting applications to that platform, especially when the application is large and complicated. Even for the more complicated OpenCL and SYCL, a new backend needs less than 400 lines of scheme code which is still very few. Secondly, once we change an existing part or add a new algorithm to the application implemented with PSCMC, they will support all the parallel platforms automatically. Last but not least, the PSCMC compiler supports generating pure serial $\mathrm{C}$ codes, which is more convenient for debugging purpose. Once the generated serial $\mathrm{C}$ code behaviors as expected but a parallel code does not, it means that errors have occurred during parallelization and thus can provide hint for debugging.

We should clarify that to achieve state-of-the-art performance, it is required to consider vendor provided specific optimizations or unique features other than the MW programming model can describe. For example, asynchronous direct memory access (DMA) on Sunway CPUs, shared memory/local memory on CUDA, HIP, OpenCL and SYCL, shuffle instructions on CUDA and HIP supported GPUs, and SIMD and prefetch instruction on modern CPUs. In the PSCMC codes, all these optimizations can be implemented by using macros similar to that in the Common Lisp. These macros can generate platform-specific code based on the choice of backnXiaond, and generate equivalent codes for other platforms.

\subsection{Process-level and thread-level parallelizations}

To achieve high scalability on large-scale heterogeneous parallel computer systems, the key is to provide enough parallelism and balanced task partition. In SymPIC, the simulation domain is decomposed into small computing blocks (CBs) using the Hilbert space filling curve. An example of a $16 \times 16$ mesh grid decomposed into $4 \times 4 \mathrm{CBs}$ by the 2nd-order Hilbert curve is shown in Fig. 4(a). In this example, there are three MPI processes in total, and the 

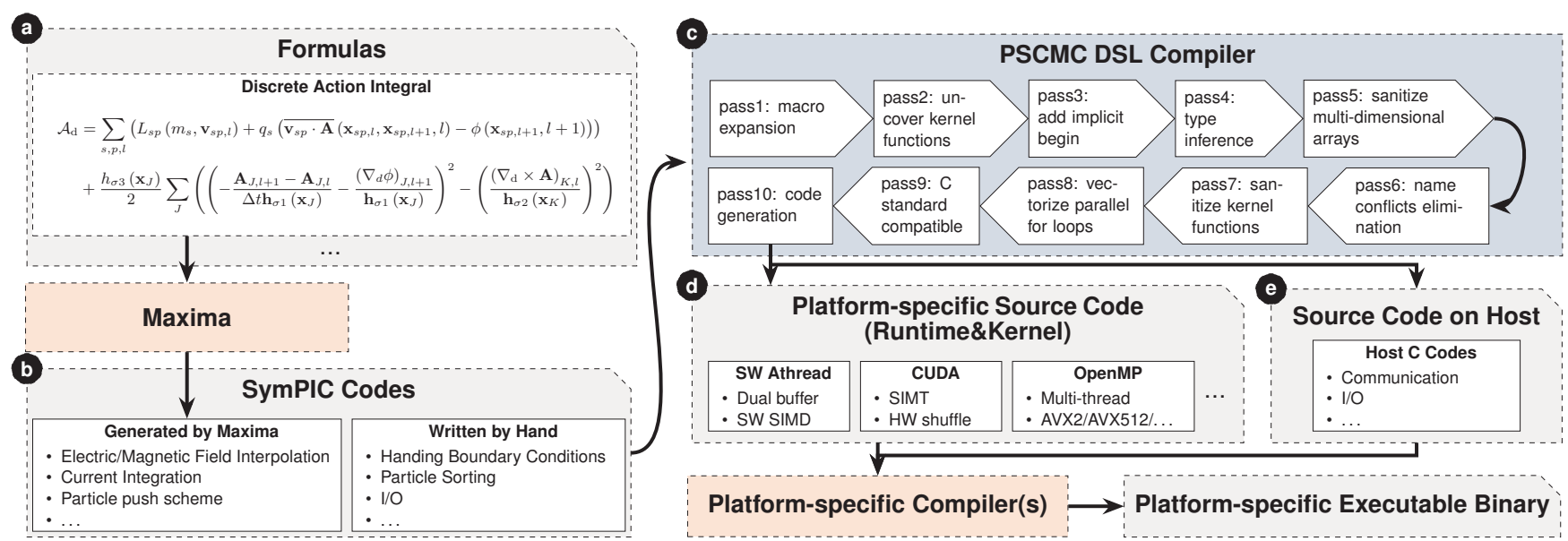

Figure 3: Typical code generation and compiling procedure of an application written in PSCMC. Here we use the SymPIC code as an example.
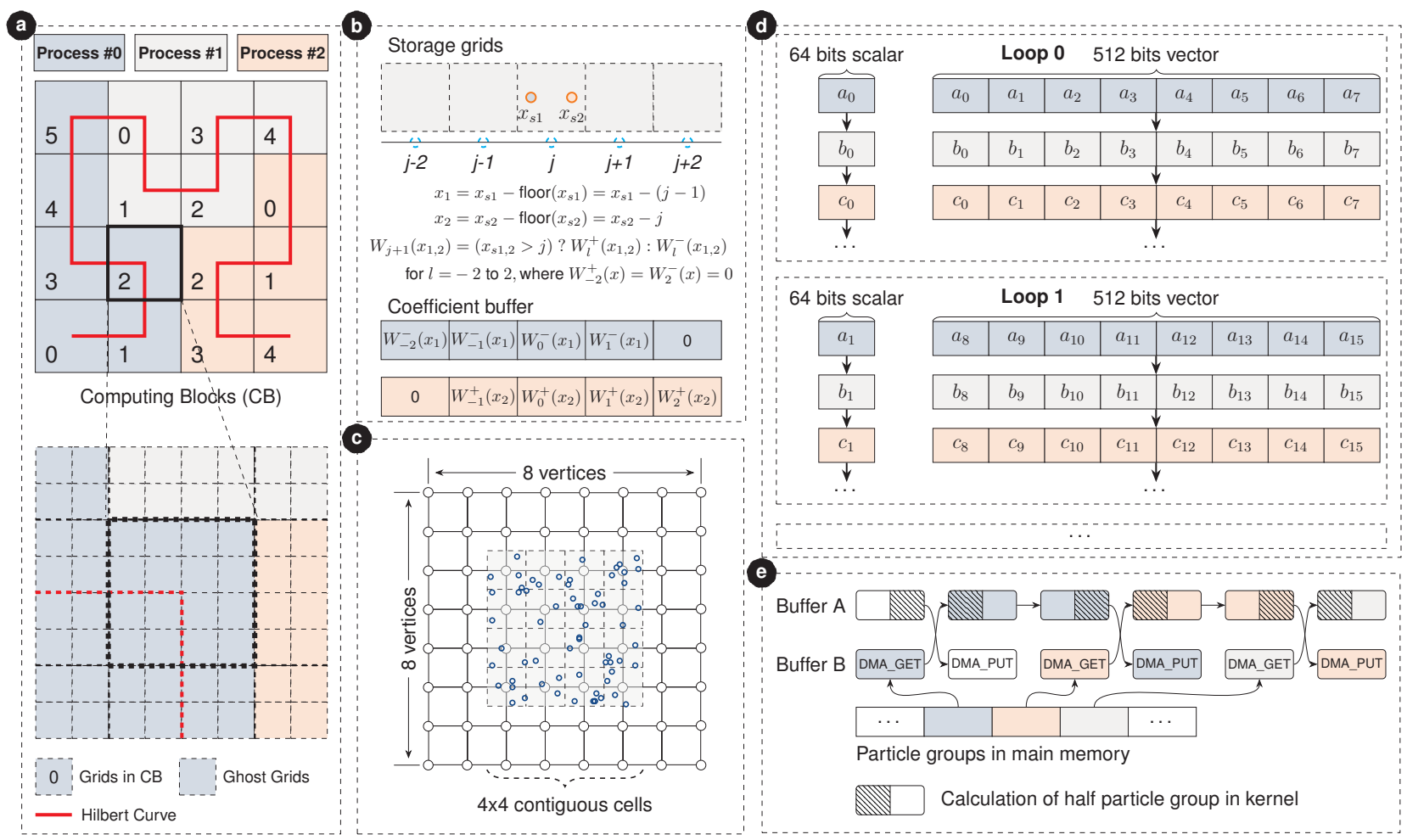

Figure 4: Parallelization scheme and optimizations in SymPIC: (a) process level parallelization; (c) branch-avoiding optimization; (d) locations of stored particles and grids; (e) auto-vectorization; (f) dual buffering optimization.

computing blocks are almost evenly distributed in the whole communication domain. Further, computing blocks can be distributed using strategies considering non-uniformly distributed particles and the performance difference of computing devices (e.g., CPU and GPU). Considering that particles can move to another grid or another $\mathrm{CB}$ during the simulation, it is necessary to get particles sorted to achieve better performance. Therefore we design a twolevel particle buffer system to handle this issue. For each grid in the $\mathrm{CB}$, a continuous memory block with fixed size, i.e., grid buffer, is allocated to store positions and velocities of particles. Typically the grid buffer size should be larger than the average number of particles in that grid. For each $\mathrm{CB}$, an additional memory block with fixed size, i.e., CB buffer, is allocated to sort particles or to store particles when overflow happens for some grids inside that $\mathrm{CB}$. In this two-level particle buffer system, most of the particles will be stored in the memory contiguously and located in the same 
nearest grid. So many advanced techniques, such as SIMD/SIMT optimization and asynchronous DMA in Sunway computing processing element (CPE), can gain more performance improvement.

We employ the 2nd-order of Whitney interpolating form in SymPIC . Therefore the knowledge of field values at $4 \times 4 \times 4$ grid points around that particle and two ghost grid layers are required for pushing one particle, as shown in Fig. 4(a). As an example, the actual grid buffer for storing field values of a $2 \mathrm{D}$ CB with size $4 \times 4$ will be $8 \times 8$. Therefore, when neighboring $\mathrm{CBs}$ update the grid data in parallel, there will be write conflicts. Adding lock can solve this problem, but this method has non-negligible time overhead on various hardware platforms. In order to generate code suitable for various platforms, SymPIC stores a data copy of ghost grids together with the local grids for each CB. This method also introduces costs when maintaining the consistency of the ghost grids. To maximize the performance, the simulation domain needs to be divided into more computing blocks to increase the parallelism, but each computing block needs to contain enough cells to keep less ghost area data copies.

To balance this trade-off, we implement two strategies for handling particle motions in the thread-level parallelism. The first CBbased task assignment strategy is that particles in one $\mathrm{CB}$ is calculated by one CPU thread (or CUDA thread-block, OpenCL workgroup, etc.), in this case there will be no conflicts between two different threads. But when the number of $\mathrm{CBs}$ in one process is small and not be a multiply of the number of hardware threads, the hardware resources will not be fully exploited. To overcome this issue, we have also implemented the second grid-based task assignment strategy. In this one all grids in one process will be most evenly distributed to CPU threads, so it will have better performance when the number of $\mathrm{CBs}$ is small compared to the first strategy. But the drawback is that an additional buffer for storing the current is required to eliminate the write conflict of each thread and an extra current accumulation procedure is called after the particle push. According to our tests when the number of CBs is a multiply of the number of CPU threads, using the first strategy the overall speed will be about $10-15 \%$ faster than using the second one.

\subsection{Particle sorting and auto-vectorization}

For modern high performance CPUs, SIMD units are usually equipped to further increase the computing density. Unfortunately until now, according to our tests modern compilers (gcc-9 and clang-10) cannot generate assembly codes that can effectively use the SIMD instruction for long loops, even with some directives such as \#pragma omp simd are given. To use these SIMD units in modern CPUs more efficiently, we design a specific syntax structure paraforn to tell the PSCMC compiler to automatically vectorize a specific for loop using specified SIMD intrinsics. The basic idea is grouping each $N_{S}$ statement then translate them into equivalent single statement using SIMD intrinsics (see Fig. $4(\mathrm{~d})$ ), where $N_{\mathrm{S}}$ is the length of SIMD vector (e.g., in the double precision case, for AVX2 $N_{\mathrm{S}}=4$, for AVX512 and Sunway 512bit SIMD $N_{\mathrm{S}}=8$ ). It currently supports all SIMD intrinsics that are supported by gcc and clang. It can also specify an SIMD mask variable to deal with the last turn of the paraforn loop where the number of rest iterations could be smaller than the length of SIMD vector.
Most of computing time in SymPIC is consumed in the particle pusher and current accumulation procedure. For particles inside one grid cell, the above procedure can be calculated together, we thus use the paraforn to automatically vectorize it. In this version of implementation, each turn $N_{S}$ particles are calculated together. It should be noted that particles will move from one grid cell to another in one time-step. To achieve better performance, the key is to eliminate branches in the algorithm. As an example, we demonstrate the calculation of interpolated electric field in one direction without introducing branch in Fig. 4(b). As we have previously mentioned, particles are mostly stored in the nearest grid point of their coordinates. So in one grid with indices $j$, the actual coordinate of the particle $x_{s p}$ should comply $j-0.5<x_{s p} \leq j+0.5$, as illustrated in Fig. 4(c). Here to simplify the notation, we let the grid size $\Delta x=1$. There are two kinds of particles, i.e., $x_{s 1}>j$ and $x_{s 2} \leq j$. Suppose we have $x_{1,2}=x_{s 1,2}-$ floor $\left(x_{s 1,2}\right)$, then their interpolation coefficients should be calculated by two divergent functions, $W_{l}^{+}$and $W_{l}^{-}$. But we can also calculate it by a predicate vector $\left(x_{s 1,2}>j\right)$, i.e.,

$$
W_{j+l}\left(x_{s 1,2}\right)=\operatorname{vselect}\left(x_{1,2}>j, W_{l}^{+}\left(x_{1,2}\right), W_{l}^{-}\left(x_{1,2}\right)\right) .
$$

If a computing device does not support the vselect instruction, it can be fallen back to

$$
\begin{aligned}
W_{j+l}\left(x_{s 1,2}\right)= & \left(x_{s 1,2}>j\right) \times W_{l}^{+}\left(x_{1,2}\right)+ \\
& \left(x_{s 1,2} \leq j\right) \times W_{l}^{-}\left(x_{1,2}\right) .
\end{aligned}
$$

Calculations of magnetic field and current can be done in a similar manner. At this point, all branches are eliminated.

Surprisingly, it should be noted that as long as the relation of $j-1 \leq x_{s p} \leq j+1$ holds, we can still correctly calculate the particle motion and current using the above procedure. Therefore the calling of the sort procedure could be less frequently than that of the calculating procedure. This further increases the overall performance a lot because the particle sorting procedure is memory bandwidth bounded. The maximum allowed number of time steps without sorting depends on the maximum speed of charged particles. Typically for electrons with thermal speed $V_{\text {th, e }}=0.05 \mathrm{c}$ and $\Delta t=0.5 \Delta x / \mathrm{c}$, we can do particle sorting once for every 4 particle pushes. One more thing, because all supported target of SymPIC share the same source code, the above branch-eliminated particle pushing code is automatically applied to the GPU version, which also improves the performance on GPUs.

\subsection{Memory-efficient optimizations}

The data required for the particle pusher and current accumulation procedure primarily includes the interpolation grids of electromagnetic fields and current, and the coordinates and velocities of the particles inside each grid in a CB. On the new Sunway supercomputer, Each CPE of the many-core CPU, SW26010Pro, has 256KB scratchpad memory (SPM) space, we configure it as $224 \mathrm{~KB}$ local data memory (LDM) and $32 \mathrm{~KB}$ cache. Therefore the LDM size is significantly larger than that of its predecessor SW26010 which only has 64KB LDM. This allows us to put more frequently accessed data into the LDM. In this work, electromagnetic fields and current in one $\mathrm{CB}$ are put in the LDM if the size of $\mathrm{CB}$ including ghost grids is smaller than a configurable number of grid points. 
In the computing kernel, particles are accessed sequentially and only once, so it is not necessary to stay in the LDM for a long time. The Sunway CPE provides an asynchronous DMA transfer mechanism to quickly load such data. To hide the memory transfer latency, the particles are grouped and loaded to two buffers in the LDM, buffer A and buffer B, as shown in Fig. 4(e). Each particle group can hold up to 32 particles. The calculation of each group is divided into two steps. In the first step, half of the group, i.e., 16 particles in buffer A are processed while the DMA_PUT operation of the particle group in buffer B is executed. And in the second step, the computation for the other 16 particles in buffer $\mathrm{A}$ is carried out while performing DMA_GET operation for loading a new particle group into buffer B. After the two steps, the pointers of buffer A and buffer B are swapped to start another cycle of the dual buffering process. On SW26010Pro, for the symplectic structure preserving PIC scheme employed in SymPIC, the time used in data transfer between LDM and main memory is smaller than that is consumed on the calculations of particles, therefore the memory transfer latency can be completely hidden.

\section{$5.6 \quad$ I/O optimizations}

In SymPIC, I/O mainly occurs when saving field results and checkpoints. During large-scale simulations, writing huge amount of data into a single file will be very time-consuming. To utilize the bandwidth provided by the filesystem in modern clusters, we design a lightweight I/O library that supports arbitrary number of I/O groups. According to our tests, in the large scale simulation of CFETR plasma with 262,144 MPI processes on the new Sunway supercomputer, $250 \mathrm{~GB}$ output data per I/O time-step can be written to the global filesystem in $1.74-10.5$ seconds when using $8192 \mathrm{I} / \mathrm{O}$ groups. The new Sunway supercomputer is also equipped with a smaller but fast object-based storage system for storing temporary data. We use it to output the checkpoints. During the above CFETR plasma simulation, one checkpoint has the size of $89 \mathrm{~TB}$. Loading or saving one such checkpoint using $32768 \mathrm{I} / \mathrm{O}$ processes will cost about 130 seconds. Usually the checkpoint is set to be updated every $1.5-2 \mathrm{hrs}$, therefore the I/O for checkpoints takes approximately $1.8 \%$ to $2.4 \%$ of the total simulation time.

\section{HOW PERFORMANCE WAS MEASURED}

\subsection{System and environment}

As the successor of Sunway TaihuLight, the new Sunway supercomputer consists of more than 103,600 SW26010Pro heterogeneous many-core processors. The architecture of SW26010Pro CPU is shown in Fig. 5. Each chip of the SW26010Pro processor contains 6 core groups (CGs). Each CG is the most basic unit that implements the manager-worker many-core architecture. In a CG, there is a management processing element (MPE) serving as the manager core and 64 computing processing elements (CPEs) arranged in an $8 \times 8$ grid acting as worker cores. As compared to its predecessor, SW26010, the upgraded CPE in SW26010Pro has 256KB scratchpad memory (SPM) and supports wider 512 bit SIMD operations. The SPM on CPE can be configured as either user-controlled local data memory (LDM) or hardware cache for automatic data buffering. The software stack on the new Sunway supercomputer includes a customized MPI library, compilers that support C, C++ and Fortran, vendor-provided library named Athread for the thread-level parallel programming on CPEs, and a job scheduler which supports management of over 40 million heterogeneous cores.

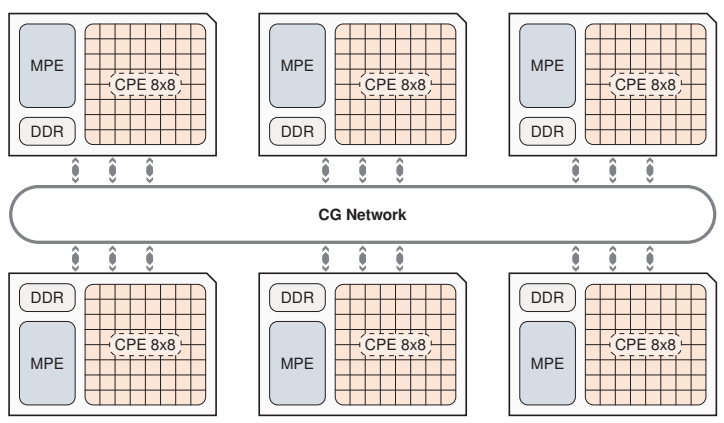

Figure 5: The architecture of SW26010Pro many-core processor equipped in the new Sunway supercomputer.

\subsection{Application description}

All tests in this article are performed on a problem that describes the toroidal plasma with following parameters unless explicitly specified,

$$
\begin{aligned}
v_{\mathrm{th}, \mathrm{e}} & =0.0138 \mathrm{c} \\
\Delta_{R} & =\Delta z=102.9 \lambda_{\mathrm{De}}, \\
\Delta_{\psi} & =3.43 \times 10^{-5} \mathrm{rad}, \\
\Delta_{\psi} R_{0} & =\Delta R, \\
\Delta_{t} & =0.5 \Delta_{R} / \mathrm{c}=\frac{0.75}{\omega_{\mathrm{pe}}}=\frac{0.59}{\omega_{\mathrm{ce}}}, \\
\mathbf{B}_{\mathrm{ext}}(R) & =\frac{R_{0} B_{0}}{R} \mathbf{e}_{\psi} .
\end{aligned}
$$

where $v_{\text {th, }}$ is the thermal velocity for electrons, $R_{0}=2920 \Delta R$ is the location of the left boundary of the simulation domain, $\omega_{\text {ce }}=$ $B_{0} \mathrm{e} / m_{\mathrm{e}}, \omega_{\text {pe }}$ and $\lambda_{\text {De }}$ are the electron plasma frequency and Debye length. These parameters can be used in simulations of wholevolume Tokamak plasmas that can resolve gyro-motion of ions. To reduce the numerical noise, the number of marker particles per grid for electrons is set to 1024 . For performance testing cases, ions are set to be fixed.

\subsection{FLOPs measurement}

To achieve high-fidelity simulations, SymPIC needs to use the double precision floating point numbers as required by the structure preserving symplectic PIC scheme. Since the generated code is very large, manually counting all double-precision arithmetic instructions in the assembly code is impractical. Instead, we use the following two methods to obtain FLOPs performance:

(1) collecting the number of double-precision arithmetic operations by using the hardware performance monitor provided by the vendor of new Sunway supercomputer,

(2) and using the Linux perf tool to measure the double-precision arithmetic operations on an Intel Xeon Gold 6142 computer 
by running the same OpenMP version of our code with AVX512 optimizations.

Result from the first method indicates that for calculating one particle, approximately $5.4 \times 10^{3}$ FLOPs is required, while the corresponding number reported by the second method is around $5.1 \times$ $10^{3}$. This slight discrepancy originates from the difference of these two platforms, and is relatively small. In this work we use the first method to calculate the FLOPs results.

\section{PERFORMANCE RESULTS}

\subsection{Portability test on various platforms}

Since our SymPIC code can be ported to many heterogeneous computing platforms, it is interesting to examine its performance on them. We list the single-node or single-device results of SymPIC to compare the performance of SymPIC on some typical platforms in Tab. 2. In the table, we summarize the running time of each PIC iteration loop to simulate a 3D computational domain with each grid containing 1024 marker particles initially. Each CB has a shape of $4 \times 4 \times 4$ and the order of 3D Hilbert curve is chosen as the maximum value allowed by the memory. "Push" means the number of million particle pushes per second for a PIC iteration step without the sort procedure, "All" means the average Push performance if one sort procedure is called every 4 PIC iterations. It can be seen from the table that the SymPIC code performs well on all the tested heterogeneous computing devices, clearly indicating that SymPIC is able to efficiently support heterogeneous computing across different architectures. In particular, with 390 cores and 512bit SIMD FPU, SW26010Pro performs the best amongst these platforms.

Table 2: Performance comparison of SymPIC on various heterogeneous platforms. Here N.C. means the number of cores. On CUDA GPUs, we record one GPU SM unit as one core, and the $64 \mathrm{bit}^{*} 32$ SIMD means each thread-block contains 32 CUDA threads. The TH2A node consists two 12-core E5-2692v2 CPUs and two 128-core Matrix-2000 accelerators, so the number of cores is 280 .

\begin{tabular}{lllllll}
\hline Hardware & ISA & Arch. & SIMD & N.C. & Push & All \\
\hline Gold 6248 & x64 & CSL & AVX512 & 40 & 220 & 192 \\
E5-2680v3 & x64 & Haswell & AVX2 & 24 & 69.8 & 65.1 \\
Hi1620-48 & ARMv8 & TS-V110 & ASIMD & 96 & 101 & 95.4 \\
Phi-7210 & x64 & KNL & AVX512 & 64 & 114.7 & 106.6 \\
Titan V & - & GV100 & 64bit*32 & 80 & 98.3 & 87.0 \\
Tesla A100 & - & GA100 & 64bit*32 & 108 & 224 & 194.4 \\
TH2A node & - & IVB+MT & AVX & 280 & 140.8 & 114.3 \\
SW26010Pro & SW & SW & 512bit & 390 & 344 & 261.1 \\
\hline
\end{tabular}

\subsection{Many-core acceleration on SW26010Pro}

To further investigate the performance improvement of SymPIC on the new Sunway platform, we compare the speed-up results using optimization techniques introduced in this work. In each CG of SW26010Pro, most computing performance is provided by the 64 CPEs. Therefore to achieve high performance on the new Sunway supercomputer, it is crucial to effectively use all these CPEs. Figure 6 shows the performance evaluation of SymPIC considering various optimizations employed in this work. The running time of the MPE-only version generated by the PSCMC serial C code backend is used as a baseline.

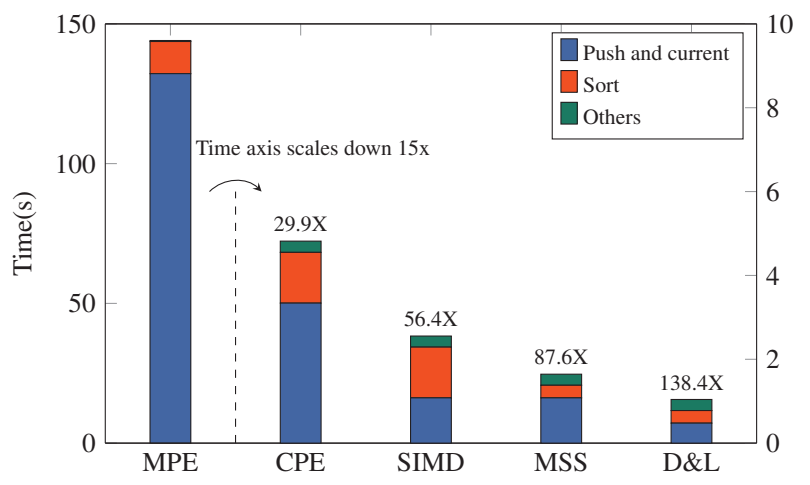

Figure 6: The running time of different subroutines in each PIC iteration loop using 8 CGs for computing problems with the configuration same to the portability tests. Here MPE, CPE, SIMD, MSS and $D \& L$ represent the MPE-only version, the initial CPE version generated by PSCMC, the 512 bit SIMD vectorized version, the multi-step sort version, and the final version including dual buffering and LDM optimizations, respectively.

For the MPE-only version, the most time-consumption kernel in SymPIC is the particle push and current calculator, which takes 91.8\% wall-time. The corresponding CPE version directly generated by PSCMC is 39.6 times faster, and 3.09 more times faster when using the PSCMC automatic SIMD loop optimization. Finally when the dual buffering and the LDM optimizations are applied, we gain another 2.26 times performance boost. In total a 277.1 times acceleration is achieved using the fully optimized CPE kernel compared to the original MPE-only version for particle push and current calculation.

The particle sorting procedure is memory bandwidth bounded, thus directly using the PSCMC generated CPE code only has a 9.5 times performance boost. However because our particle push and current calculator allows particles that are not exactly located in the corresponding grid, the number of required sort procedure can be reduced. In this case we sort once for each 4 PIC iteration timesteps, which immediately results a 4 times acceleration. So the overall many-core acceleration of particle sort procedure is 38.0.

Combining all the optimization techniques listed above, we obtain a final 138.4 times acceleration of our symplectic PIC scheme using the Sunway CPEs.

\subsection{Strong scaling test}

In the strong scaling tests, we set the number of grids in each direction and the number of particles as in Tab. 3. The size of one CB is $4 \times 4 \times 6$ and the sort procedure is called every 4 iteration steps, the I/O module of SymPIC is disabled, and only the PIC iteration time is recorded. Performances are calculated using the wall time for iterating 100 time-steps.

The measured strong scalability results are shown in Fig. 7. Here for problem $A$, because the number of CBs is $2^{24}$, so when the total numbers of CPEs are larger than $2^{24}$ (for 524,288 and 616,200 CGs) 
Table 3: Number of grids and particles for the strong scaling test, where two scales (A and B) of problems are tested.

\begin{tabular}{lrrrrr}
\hline Scale & $N_{R}$ & $N_{\psi}$ & $N_{z}$ & \# marker particles & Number of CGs \\
\hline A & 1024 & 1024 & 1536 & $1.65 \times 10^{12}$ & 616200 \\
A & 1024 & 1024 & 1536 & $1.65 \times 10^{12}$ & 524288 \\
A & 1024 & 1024 & 1536 & $1.65 \times 10^{12}$ & 262144 \\
A & 1024 & 1024 & 1536 & $1.65 \times 10^{12}$ & 131072 \\
A & 1024 & 1024 & 1536 & $1.65 \times 10^{12}$ & 65536 \\
A & 1024 & 1024 & 1536 & $1.65 \times 10^{12}$ & 32768 \\
A & 1024 & 1024 & 1536 & $1.65 \times 10^{12}$ & 16384 \\
B & 2048 & 2048 & 3072 & $1.32 \times 10^{13}$ & 616200 \\
B & 2048 & 2048 & 3072 & $1.32 \times 10^{13}$ & 524288 \\
B & 2048 & 2048 & 3072 & $1.32 \times 10^{13}$ & 262144 \\
B & 2048 & 2048 & 3072 & $1.32 \times 10^{13}$ & 131072 \\
\hline
\end{tabular}

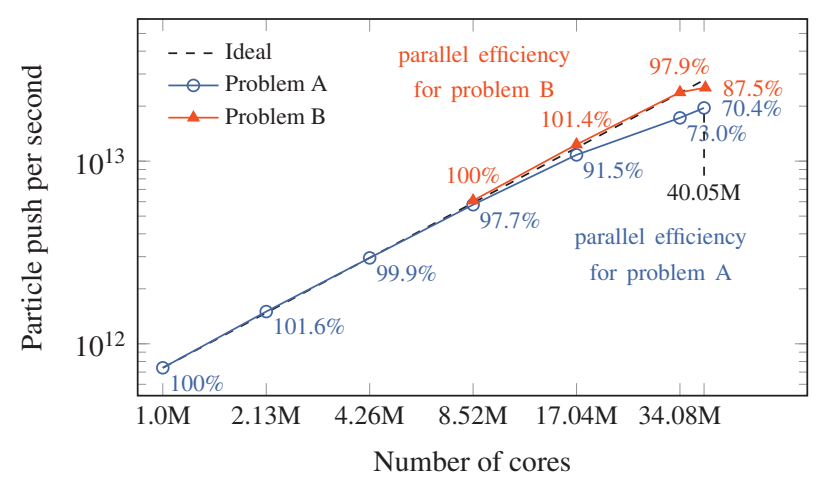

Figure 7: Sustained performance of the strong scaling test.

we use the grid-based discretization strategy for updating particles, which increases the total computational complexity but provides more parallelism; for other cases we still use the CB-based discretization strategy. It can be seen that SymPIC has a good strong scaling efficiency of $91.5 \%$ when the number of CGs increases from 16,384 to 262,144 . For 524,288 and 616,200 CGs because of the adoption of the grid-based discretization strategy the parallel efficiency drops to $73.0 \%$ and $70.4 \%$. But it is still better than using CB-based one because in these cases the particle push procedure using CBbased discretization strategy cannot gain performance compared with using 262,144 CGs. For problem B which is around 8 times larger than problem A, scaling from 131,072 to 524,288 CGs leads to a parallel efficiency of $97.9 \%$. For the largest case of 616,200 CGs we have tested both CB-based and grid-based strategies and find that the CB-based one performs better. So the overall strong scaling efficiency for problem B from 131,072 CGs $(8,519,680$ processor cores) to 616,200 CGs $(40,053,000$ processor cores $)$ is $87.5 \%$.

\subsection{Weak scaling test}

In the weak scaling tests, the configurations of the problem is shown in Tab. 4, and the experiments are done following similar rules as in the strong scaling tests. The test results are presented in Fig. 8, from which it can be seen that the performance of SymPIC increases nearly perfectly as the number of CGs and problem scale
Table 4: Number of grids and particles for weak scaling tests.

\begin{tabular}{rrrrr}
\hline$N_{R}$ & $N_{\psi}$ & $N_{z}$ & \# marker particles & Number of CGs \\
\hline 64 & 64 & 96 & $4.03 \times 10^{8}$ & 8 \\
128 & 128 & 192 & $3.22 \times 10^{9}$ & 64 \\
256 & 256 & 384 & $2.58 \times 10^{10}$ & 512 \\
512 & 512 & 768 & $2.06 \times 10^{11}$ & 4096 \\
1024 & 1024 & 1536 & $1.65 \times 10^{12}$ & 32768 \\
2048 & 2048 & 3072 & $1.32 \times 10^{13}$ & 262144 \\
3072 & 2048 & 4096 & $2.64 \times 10^{13}$ & 621600 \\
\hline
\end{tabular}

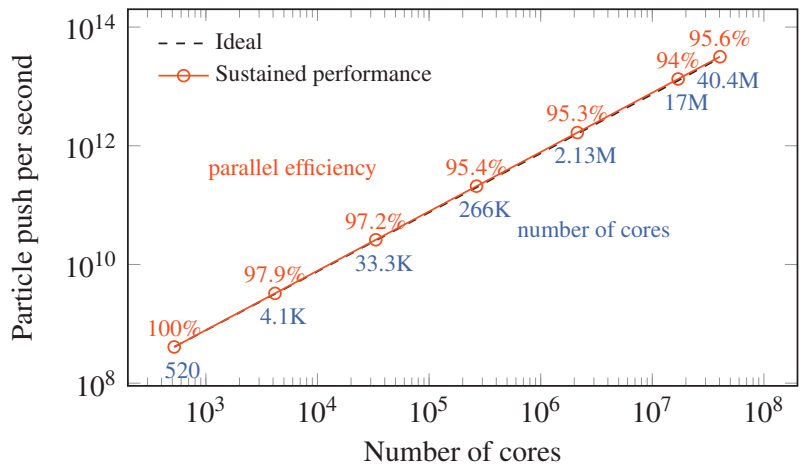

Figure 8: Sustained performance of the weak scaling test.

increases. We obtain a weak scaling efficiency of $95.6 \%$ from 8 CGs (520 processor cores) to 621,600 CGs $(40,404,000$ processor cores), corresponding number of particles and grids are from $4.03 \times 10^{8}$ to $2.639 \times 10^{13}$ and $3.93 \times 10^{5}$ to $2.577 \times 10^{10}$, respectively.

\subsection{Peak performance test}

Table 5: Number of grids and particles for the peak performance test.

\begin{tabular}{rrrrr}
\hline$N_{R}$ & $N_{\psi}$ & $N_{z}$ & \# marker particles & Number of CGs \\
\hline 3072 & 2048 & 4096 & $1.113 \times 10^{14}$ & 621600 \\
\hline
\end{tabular}

For peak performance measurement, we use the simulation parameters displayed in the Tab. 5. We use the the plasma of $3072 \times$ $2048 \times 4096$ grids with 4320 electron marker particles per cell, and the simulation is performed on 103,600 nodes $(40,404,000$ processor cores) of the new Sunway supercomputer. The sorting procedure is called every 4 time-steps. For one iteration time-step without sort procedure, 2.016 second is cost which resulting a peak performance of $298.2 \mathrm{PFLOP} / \mathrm{s}$ in double precision. For each 4 time-step iterations, additional 3.890 second is cost to call the sort procedure, therefore the average iteration time for one time-step is $2.989 \mathrm{sec}-$ ond and we can obtain a sustained performance of $201.1 \mathrm{PFLOP} / \mathrm{s}$ in double precision. The code reaches a final $3.724 \times 10^{13}$ sustained particle push per second performance. 


\section{IMPLICATIONS}

\subsection{Application results}

Using the optimized SymPIC code on the new Sunway supercomputer, we have managed to simulate two whole-volume Tokamak $\mathrm{H}$-mode plasmas using 2D fluid equilibrium profiles.

The first one is based on the equilibrium of $\mathrm{H}$-mode plasma generated in the shot-86541 of EAST, its resolution is $N_{R} \times N_{\psi} \times N_{z}=$ $768 \times 256 \times 768$ with $\Delta R \approx 0.55 \rho_{i}$ where $\rho_{i}$ is the gyro-radius of thermal ions. Here electron-deuterium plasma is used, the mass ratio between electron and deuterium is $1: 200$, and in the center of the plasma NPG of electrons and ions are set to 768 and 128. In this case 32768 CGs $(2,129,920$ processor cores) are used and the total simulation time is 1 day (including $\mathrm{I} / \mathrm{O}$, checkpoint and rerun due to the arrangement of job queue). A total $3.4 \times 10^{5}$ time-steps are simulated, and the 3D density distribution of last time-step can be shown in Fig. 9 (a). From Fig. 9 (a) we can see that at the edge of the plasma belt-structure unstable modes occurs. Structures of unstable modes with different toroidal mode number $n$ can be also demonstrated in Fig. 9 (b).

For the second case, we generated a plasma based on the fluid equilibrium of $\mathrm{H}$-mode burning plasma in a designed fusion operation mode of CFETR. Its resolution is $N_{R} \times N_{\psi} \times N_{z}=1024 \times$ $512 \times 1024$ with $\Delta R \approx 1.5 \rho_{i}$ where $\rho_{i}$ is the gyro-radius of thermal deuteriums. This plasma containes 7 species of charged particles, which are electrons with mass of 73.44 times the real mass of electrons, deuteriums, tritiums, thermal heliums, argons, fast deuteriums with a center temperature of $200 \mathrm{keV}$, energetic fusions generated alpha particle with a center temperature of $1081 \mathrm{keV}$. Corresponding NPG in the center for each species are set to $768,52,52$, $10,10,10,80$. In this case 262,144 CGs (17,039,360 processor cores) are used and the total simulation time is 1.5 days (including $\mathrm{I} / \mathrm{O}$, checkpoint, and rerun due to the node failure). A total $4.6 \times 10^{5}$ time-steps are simulated, and the 3D pressure of last time-step can be shown in Fig. 10 (a). It is clear that the designed CFETR H-mode plasma is much more stable than the EAST H-mode plasma, and we can barely see the unstable modes from the density perturbation. To demonstrate the unstable modes at the edge, we also draw the magnetic perturbation of different toroidal mode number $n$ in Fig. 10 (b). For the first time, the unprecedented whole-volume fusion plasma containing deuteriums, tritiums, heliums, fast deuteriums and fusion generated energetic alpha particles are directly simulated with the electromagnetic fully kinetic PIC method. Because of the excellent conservation property of the symplectic PIC scheme, we can investigate the low frequency phenomena in the plasma without worrying about the numerical dissipation problems.

\subsection{Symplectic structure-preserving electromagnetic fully kinetic PIC simulation of whole-volume Tokamak plasmas}

We have implemented and optimized our recently developed symplectic structure-preserving PIC scheme for cylindrical regular meshes on the Sunway many-core architecture. With the help of world's leading new Sunway supercomputer, unprecedented large-scale
Tokamak plasmas can be directly simulated by using the fully kinetic PIC method. This enables the numerical investigation of many crucial physical and engineering problems for whole-volume Tokamak plasmas using the fully kinetic model. Compared to gyrokinetic PIC schemes used previously, the present method is based on a more fundamental Vlasov-Maxwell physical model. A typical advantage in Tokamak physics is that in our simulations the selfconsistent electric field is naturally formed in the $2 \mathrm{D}$ equilibrium of plasma, and it evolves nonlinearly with the evolution of the 6D whole-volume plasma. This is crucial in Tokamak plasmas especially when investigating edge related physics since at that place radial electric fields are strong and has close relation to $\mathbf{E} \times \mathbf{B}$ drift motion, instabilities and turbulences.

In this work, the most unstable mode of whole-volume Tokamak plasma can be obtained and nextly we can try to find a way to suppress it, which may serve as a guidance to improve the performance of future large Tokamaks such as ITER and CFETR, and eventually accomplish more economical controlled fusion energy. Other important problems such as wave heating and current drive, energetic particles induced instabilities, energy deposition of fusion alpha particles can be also investigated. Before the machines are actually built, fully kinetic simulation results will also support and improve their designs.

\subsection{SymPIC and PSCMC on other platforms}

Though this work mainly focus on the optimization of SymPIC on the Sunway many-core platform, it should be noted that SymPIC has excellent portability because the employment of PSCMC and most performance optimizations introduced in this work can be directly applied to other hardware architectures. For example the automatic SIMD loop optimizer can generate any SIMD intrinsics that supported by gec or clang and hence boost the performance on common high-performance CPU platforms, while the reduction of number of sort procedure will also reduce the memory bandwidth requirement on conventional multi-core CPU and GPU nodes. These optimizations have already applied to SymPIC on other heterogeneous platforms.

The PSCMC can be also employed to implement other applications or algorithms that can beneficial from the managementworker programming model. It makes single-source code that supports all present world's leading supercomputers possible. The most exciting part is that writing a PSCMC backend to support new heterogeneous platform requires very small effort. In most cases it is much easier than directly porting the applications to support that platform. While platform specific optimizations can be also implemented by writing macros in PSCMC source code. This means both high efficiency and high portability can be achieved together, which is highly desired for today's high performance computing developments. We have open sourced both SymPIC and PSCMC, and hope that our efforts will be of common interest to the community of PIC simulations and Tokamak plasma physics.

\section{ACKNOWLEDGEMENT}

This work was supported by the the National MCF Energy R\&D Program (2018YFE0304100, 2019YFE03090200), National Key Research and Development Program (2016YFA0400600, 2016YFA0400601 and 2016YFA0400602), 

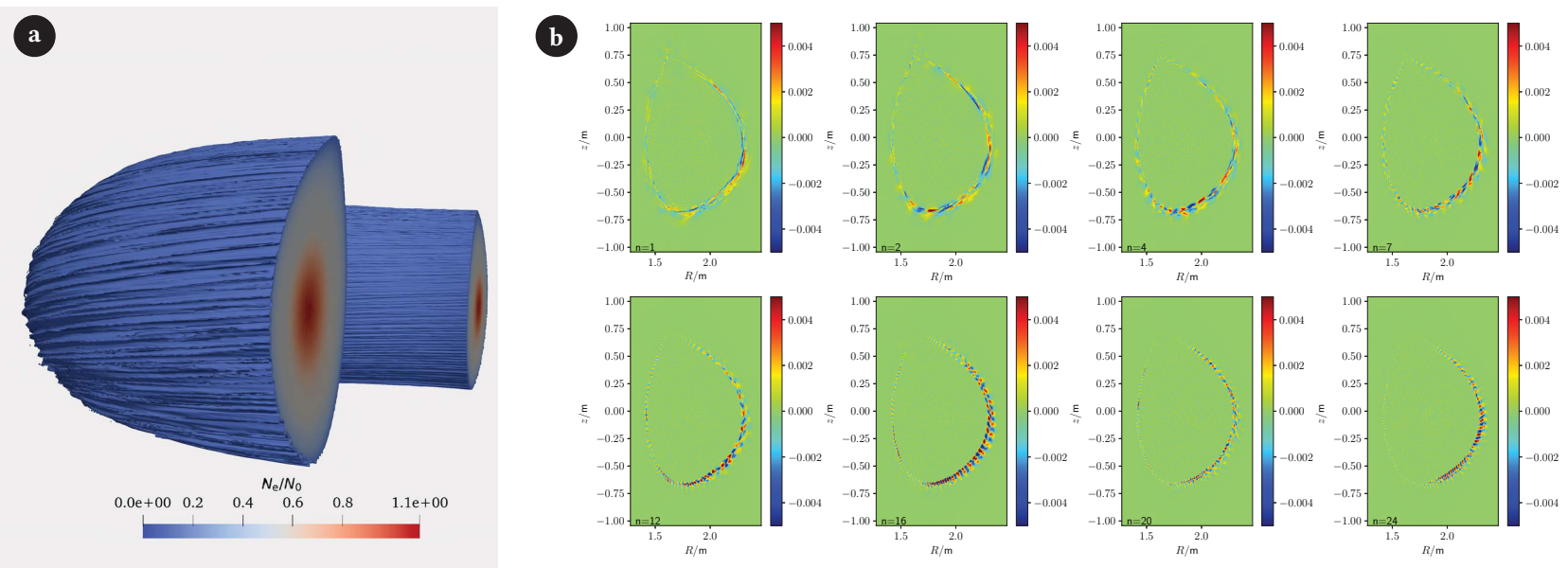

Figure 9: Simulation results of the EAST H-mode plasma. (a) The 3D density distribution of a turbulence state. (b) The unstable mode structures of electron density with different toroidal mode number $n$. Here the contour shows mode distribution normalized by the initial core density $n_{0}$.
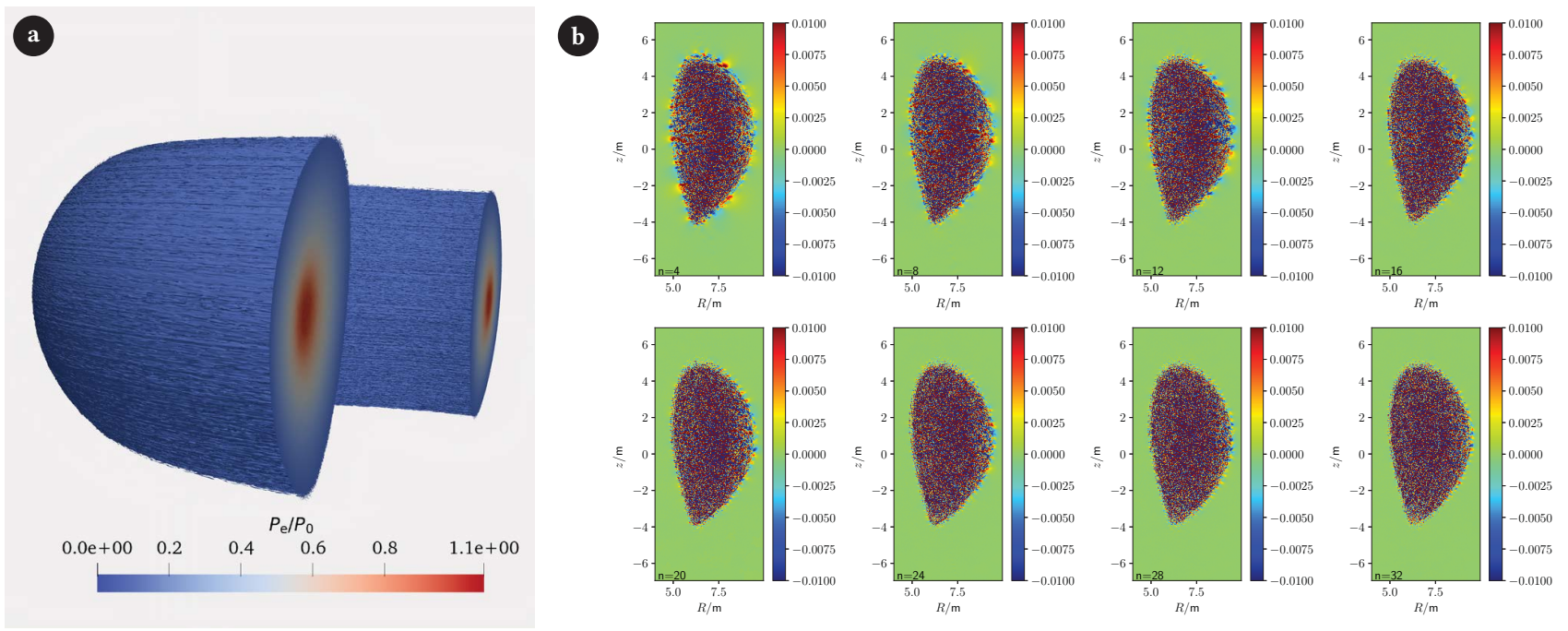

Figure 10: Simulation results of the designed CFETR H-mode plasma. (a) The 3D pressure distribution of a instability increasing state. (b) The unstable mode structures of $B_{R}$ with different toroidal mode number $n$. Here the contour shows mode distribution normalized by 1 Tesla. It can be seen that the unstable mode also occurs at the edge of the plasma.

and the National Natural Science Foundation of China (NSFC-11905220, 11805273 and 11975230). Small scale performance evaluation results of x86 and ARM based CPUs, Tesla A100 GPUs and NUDT matrix accelerators were carried out at Supercomputing Center of the University of Science and Technology of China and TianHe-2A in the National Supercomputer Center in Guangzhou. J. Xiao would like to thank Dr. Peifeng Fan at Shenzhen University for the valuable discussions on gyrokinetic physical models.

\section{REFERENCES}

[1] [n.d.]. Maxima, a Computer Algebra System. https://maxima.sourceforge.io/. Accessed: 2021-08-02.

[2] LA Artsimovich. 1972. Tokamak devices. Nuclear Fusion 12, 2 (1972), 215.

[3] R Aymar, P Barabaschi, and Y Shimomura. 2002. The ITER design. Plasma Physics and Controlled Fusion 44, 5 (apr 2002), 519-565. https://doi.org/10.1088/0741$3335 / 44 / 5 / 304$
[4] Robert Bird, Nigel Tan, Scott V Luedtke, Stephen Lien Harrell, Michela Taufer, and Brian Albright. 2021. VPIC 2.0: Next Generation Particle-in-Cell Simulations. arXiv preprint arXiv:2102.13133 (2021).

[5] Charles K Birdsall. 1991. Particle-in-cell charged-particle simulations, plus Monte Carlo collisions with neutral atoms, PIC-MCC. IEEE Transactions on plasma science 19, 2 (1991), 65-85. https://doi.org/10.1109/27.106800

[6] Charles K Birdsall and A Bruce Langdon. 1991. Plasma Physics via Computer Simulation. IOP Publishing. 293 pages.

[7] Paul Bonoli, Lois Curfman McInnes, C Sovinec, D Brennan, T Rognlien, P Snyder, J Candy, C Kessel, J Hittinger, L Chacon, et al. 2015. Report of the workshop on integrated simulations for magnetic fusion energy sciences. Technical Report. US Department of Energy Office of Science, Office of Fusion Energy Scienced and Office of Advanced Scientific Computing Research, Tech. Rep.

[8] K. J. Bowers, B. J. Albright, B. Bergen, L. Yin, K. J. Barker, and D. J. Kerbyson. 2008. 0.374 Pflop/s trillion-particle kinetic modeling of laser plasma interaction on roadrunner. In SC '08: Proceedings of the 2008 ACM/IEEE Conference on Supercomputing. 1-11. https://doi.org/10.1109/SC.2008.5222734 
[9] K. J. Bowers, B. J. Albright, L. Yin, B. Bergen, and T. J. T. Kwan. 2008. Ultrahigh performance three-dimensional electromagnetic relativistic kinetic plasma simulation. Physics of Plasmas 15, 5 (2008), 055703. https://doi.org/10.1063/1 2840133 arXiv:https://doi.org/10.1063/1.2840133

[10] M. Bussmann, H. Burau, T. E. Cowan, A. Debus, A. Huebl, G. Juckeland, T. Kluge, W. E. Nagel, R. Pausch, F. Schmitt, U. Schramm, J. Schuchart, and R. Widera. 2013 Radiative signature of the relativistic Kelvin-Helmholtz Instability. In SC '13: Proceedings of the International Conference on High Performance Computing, Networking, Storage and Analysis. 1-12. https://doi.org/10.1145/2503210.2504564

[11] S. Chandrasekaran, A. Debus, T. Kluge, R. Widera, S. Bastrakov, K. Steiniger, M Garten, M. Werner, J. Kelling, M. Leinhauser, J. Young, J. H. Davis, J. M. Diaz, B. Worpitz, A. Huebl, R. Chatterjee, G. Juckeland, and M. Bussmann. 2019. Running PIConGPU on Summit CAAR: Preparing PIConGPU for Frontier @ ORNL. In SC '19: Proceedings of the 2019 ACM/IEEE Conference on Supercomputing.

[12] C. S. Chang, S. Ku, G. R. Tynan, R. Hager, R. M. Churchill, I. Cziegler, M. Green wald, A. E. Hubbard, and J. W. Hughes. 2017. Fast Low-to-High Confinement Mode Bifurcation Dynamics in a Tokamak Edge Plasma Gyrokinetic Simulation. Phys. Rev. Lett. 118 (Apr 2017), 175001. Issue 17. https://doi.org/10.1103/ PhysRevLett.118.175001

[13] John M Dawson. 1983. Particle simulation of plasmas. Reviews of Modern Physics 55, 2 (1983), 403. https://doi.org/10.1103/RevModPhys.55.403

[14] E. A. Frieman, R. C. Davidson, and B. Langdon. 1966. Higher-Order Corrections to Chew-Goldberger-Low Theory. Physics of Fluids 9, 8 (1966), 1475-1482.

[15] T. S. Hahm, W. W. Lee, and A. Brizard. 1988. Nonlinear gyrokinetic theory for finite-beta plasmas. The Physics of Fluids 31, 7 (1988), 1940-1948. https://doi org/10.1063/1.866641 arXiv:https://aip.scitation.org/doi/pdf/10.1063/1.866641

[16] Yang He, Hong Qin, Yajuan Sun, Jianyuan Xiao, Ruili Zhang, and Jian Liu. 2015 Hamiltonian time integrators for Vlasov-Maxwell equations. Physics of Plasmas (1994-present) 22, 12 (2015), 124503. https://doi.org/10.1063/1.4938034

[17] Eero Hirvijoki, Katharina Kormann, and Filippo Zonta. 2020. Subcycling of particle orbits in variational, geometric electromagnetic particle-in-cell methods. Physics of Plasmas 27, 9 (2020), 092506. https://doi.org/10.1063/5.0006403 arXiv:https://doi.org/10.1063/5.0006403

[18] R.W Hockney. 1971. Measurements of collision and heating times in a twodimensional thermal computer plasma. 7. Comput. Phys. 8, 1 (1971), $19-44$ https://doi.org/10.1016/0021-9991(71)90032-5

[19] Roger W Hockney and James W Eastwood. 1988. Computer Simulation Using Particles. CRC Press.

[20] Youjun Hu, Matthew T Miecnikowski, Yang Chen, and Scott E Parker. 2018 Fully Kinetic Simulation of Ion-Temperature-Gradient Instabilities in Tokamaks. Plasma 1, 1 (2018), 105-118. https://doi.org/10.3390/plasma1010010

[21] Andrew W. Keep and R. Kent Dybvig. 2013. A Nanopass Framework for Commercial Compiler Development. SIGPLAN Not. 48, 9 (Sept. 2013), 343-350. https://doi.org/10.1145/2544174.2500618

[22] Michael Kraus, Katharina Kormann, Philip J. Morrison, and Eric Sonnendrücker 2017. GEMPIC: geometric electromagnetic particle-in-cell methods. four nal of Plasma Physics 83, 4 (jul 2017), 905830401. https://doi.org/10.1017/ s002237781700040x

[23] WW Lee. 1987. Gyrokinetic particle simulation model. F. Comput. Phys. 72, 1 (1987), 243-269.

[24] W. W. Lee. 1983. Gyrokinetic approach in particle simulation. The Physics of Fluids 26, 2 (1983), 556-562. https://doi.org/10.1063/1.864140 arXiv:https://aip.scitation.org/doi/pdf/10.1063/1.864140

[25] Zhihong Lin, Taik Soo Hahm, WW Lee, William M Tang, and Roscoe B White. 1998. Turbulent transport reduction by zonal flows: Massively parallel simulations. Science 281, 5384 (1998), 1835-1837. https://doi.org/10.2172/289931

[26] Matthew T. Miecnikowski, Benjamin J. Sturdevant, Yang Chen, and Scott E Parker. 2018. Nonlinear saturation of the slab ITG instability and zonal flow generation with fully kinetic ions. Physics of Plasmas 25, 5 (2018), 055901. https://doi.org/10.1063/1.5011681 arXiv:https://doi.org/10.1063/1.5011681

[27] P. J. Morrison. 2017. Structure and structure-preserving algorithms for plasma physics. Physics of Plasmas 24, 5 (apr 2017), 055502. https://doi.org/10.1063/1. 4982054

[28] Noé Ohana, Claudio Gheller, Emmanuel Lanti, Andreas Jocksch, Stephan Brunner, and Laurent Villard. 2021. Gyrokinetic simulations on many- and multi-core architectures with the global electromagnetic Particle-In-Cell Code ORB5. Com puter Physics Communications 262 (2021), 107208. https://doi.org/10.1016/j.cpc. 2020.107208

[29] Hong Oin, Jian Liu, Jianyuan Xiao, Ruili Zhang, Yang He, Yulei Wang, Yajuan Sun, Joshua W. Burby, Leland Ellison, and Yao Zhou. 2016. Canonical symplectic particle-in-cell method for long-term large-scale simulations of the VlasovMaxwell equations. Nuclear Fusion 56, 1 (2016), 014001. https://doi.org/10.1088/ 0029-5515/56/1/014001

[30] P. Rodriguez-Fernandez, N. T. Howard, M. J. Greenwald, A. J. Creely, J. W. Hughes, J. C. Wright, C. Holland, Y. Lin, and F. Sciortino. 2020. Predictions of core plasma performance for the SPARC tokamak. Fournal of Plasma Physics 86, 5 (2020), 865860503. https://doi.org/10.1017/S0022377820001075
[31] Dipanwita Sarkar, Oscar Waddell, and R. Kent Dybvig. 2005. EDUCATIONAL PEARL: A Nanopass framework for compiler education. Fournal of Functional Programming 15, 5 (2005), 653-667. https://doi.org/10.1017/S0956796805005605

[32] Jonathan Squire, Hong Oin, and William M Tang. 2012. Geometric integration of the Vlasov-Maxwell system with a variational particle-in-cell scheme. Physics of Plasmas 19, 8 (2012), 084501. https://doi.org/10.1063/1.4742985

[33] D Tskhakaya, K Matyash, R Schneider, and F Taccogna. 2007. The Particle-InCell Method. Contributions to Plasma Physics 47, 8-9 (2007), 563-594.

[34] Weigang Wan, Scott E Parker, Yang Chen, Zheng Yan, Richard J Groebner, and Philip B Snyder. 2012. Global gyrokinetic simulation of tokamak edge pedestal instabilities. Physical review letters 109, 18 (2012), 185004. https://doi.org/10. 1103/physrevlett.109.185004

[35] Bei Wang, Stephane Ethier, William Tang, Khaled Z Ibrahim, Kamesh Madduri, Samuel Williams, and Leonid Oliker. 2019. Modern gyrokinetic particle-in-cell simulation of fusion plasmas on top supercomputers. The International fournal of High Performance Computing Applications 33, 1 (2019), 169-188. https://doi. org/10.1177/1094342017712059 arXiv:https://doi.org/10.1177/1094342017712059

[36] Bei Wang, Stephane Ethier, William Tang, Timothy Williams, Khaled Z Ibrahim, Kamesh Madduri, Samuel Williams, and Leonid Oliker. 2013. Kinetic turbulence simulations at extreme scale on leadership-class systems. In Proceedings of the International Conference on High Performance Computing, Networking, Storage and Analysis. 1-12. https://doi.org/10.1145/2503210.2503258

[37] Songtao Wu. 2007. An overview of the EAST project. Fusion Engineering and Design 82, 5 (2007), 463-471. https://doi.org/10.1016/j.fusengdes.2007.03.012 Proceedings of the 24th Symposium on Fusion Technology.

[38] Samuel E Wurzel and Scott C Hsu. 2021. Progress toward Fusion Energy Breakeven and Gain as Measured against the Lawson Criterion. arXiv preprint $\operatorname{arXiv:2105.10954(2021)}$

[39] Jianyuan Xiao, Jian Liu, Hong Oin, and Zhi Yu. 2013. A variational multisymplectic particle-in-cell algorithm with smoothing functions for the VlasovMaxwell system. Physics of Plasmas 20, 10 (oct 2013), 102517. https://doi.org/ $10.1063 / 1.4826218$

[40] Jianyuan Xiao and Hong Oin. 2019. Field theory and a structure-preserving geometric particle-in-cell algorithm for drift wave instability and turbulence. $\mathrm{Nu}$ clear Fusion 59, 10 (sep 2019), 106044. https://doi.org/10.1088/1741-4326/ab38dc

[41] Jianyuan Xiao and Hong Qin. 2021. Explicit structure-preserving geometric particle-in-cell algorithm in curvilinear orthogonal coordinate systems and its applications to whole-device 6D kinetic simulations of tokamak physics. Plasma Science and Technology 23, 5 (apr 2021), 055102. https://doi.org/10.1088/2058$6272 /$ abf 125

[42] Jianyuan Xiao, Hong Qin, and Jian Liu. 2018. Structure-preserving geometric particle-in-cell methods for Vlasov-Maxwell systems. Plasma Science and Technology 20, 11, Article 110501 (2018), 0 pages. https://doi.org/10.1088/2058$6272 / \mathrm{aac} 3 \mathrm{~d} 1$

[43] Jianyuan Xiao, Hong Qin, Jian Liu, Yang He, Ruili Zhang, and Yajuan Sun. 2015. Explicit high-order non-canonical symplectic particle-in-cell algorithms for Vlasov-Maxwell systems. Physics of Plasmas 22, 11 (nov 2015), 112504. https://doi.org/10.1063/1.4935904

[44] Y Yang, X Gao, H Q Liu, G Q Li, T Zhang, L Zeng, Y K Liu, M Q Wu, D F Kong, T F Ming, X Han, Y M Wang, Q Zang, B Lyu, Y Y Li, Y M Duan, F B Zhong, K Li, L O Xu, X Z Gong, Y W Sun, J P Oian, B J Ding, Z X Liu, F K Liu, C D Hu, N Xiang, Y F Liang, X D Zhang, B N Wan, J G Li, and Y X Wan and. 2017. Observation of internal transport barrier in ELMy H-mode plasmas on the EAST tokamak. Plasma Physics and Controlled Fusion 59, 8 (jun 2017), 085003. https: //doi.org/10.1088/1361-6587/aa6f2a

[45] Erik Zenker, René Widera, Axel Huebl, Guido Juckeland, Andreas Knüpfer, Wolfgang E. Nagel, and Michael Bussmann. 2016. Performance-Portable Many-Core Plasma Simulations: Porting PIConGPU to OpenPower and Beyond. In High Performance Computing, Michela Taufer, Bernd Mohr, and Julian M. Kunkel (Eds.). Springer International Publishing, Cham, 293-301.

[46] Wenlu Zhang, Wayne Joubert, Peng Wang, Bei Wang, William Tang, Matthew Niemerg, Lei Shi, Sam Taimourzadeh, Jian Bao, and Zhihong Lin. 2019. Heterogeneous Programming and Optimization of Gyrokinetic Toroidal Code Using Directives. In Accelerator Programming Using Directives, Sunita Chandrasekaran, Guido Juckeland, and Sandra Wienke (Eds.). Springer International Publishing, Cham, 3-21.

[47] G. Zhuang, G.Q. Li, J. Li, Y.X. Wan, Y. Liu, X.L. Wang, Y.T. Song, V. Chan, Q.W. Yang, B.N. Wan, X.R. Duan, P. Fu, and B.J. Xiao and. 2019. Progress of the CFETR design. Nuclear Fusion 59, 11 (jun 2019), 112010. https://doi.org/10.1088/17414326/ab0e27 